\title{
Bringing Light to Science Undergraduate Students: A Successful Laboratory Experiment Illustrating the Principles and Applications of Bioluminiscence
}

Ángel Luis García-Ponce ${ }^{\dagger}$, José Antonio Torres-Vargas $¥$, Melissa García-Caballero $¥$, Miguel Ángel

Medina ${ }^{\ddagger}$, Ángel Blanco-López ${ }^{\dagger}$, Ana R. Quesada*‡

†Universidad de Málaga, Andalucía Tech, Didáctica de las Ciencias Experimentales, Facultad de Ciencias de la Educación. Málaga (Spain)

¥Universidad de Málaga, Andalucía Tech, Departamento Biología Molecular y Bioquímica, Facultad de Ciencias, Málaga (Spain)

\section{ABSTRACT}

Although there are many laboratory experiments available to illustrate spectrophotometric or fluorometric methods, few of them introduce the use of luminometry to students. Bioluminiscence, a subtype of chemiluminescence, is produced when an enzyme-catalyzed chemical reaction gives rise to a light emission. Despite the advantages of bioluminescent methods, including sensitivity and specificity, and their increasing use in experimental sciences and biomedical laboratories, their presence in our classrooms are almost inexistent. The luciferase-catalyzed enzymatic reaction has generated a myriad of practical applications, including those derived from the measurement of the ATP consumed in the reaction. In particular, the measurement of the ATP levels in drinking or stored waters directly correlates with their bacteria content, facilitating the development of rapid methods for detecting bacterial contamination. They avoid the long waiting time associated with the traditional microbiological methods, based on the growth of the microorganisms in a suitable culture medium. Throughout the last two years, we have implemented at the University of Malaga a new laboratory experiment for undergraduate chemistry and biochemistry students. In this experiment, students detected bacterial contamination in water by quantifying ATP with the luciferase catalyzed reaction. The experiment was implemented in two different formats, either as a full project developed by students throughout the entire duration of the academic course, or as a shorter version that could be carried out in a single laboratory session. Its application to our classrooms has been very satisfactory, as evidenced by the good academic performance and positive students' perception. 


\section{GRAPHICAL ABSTRACT}

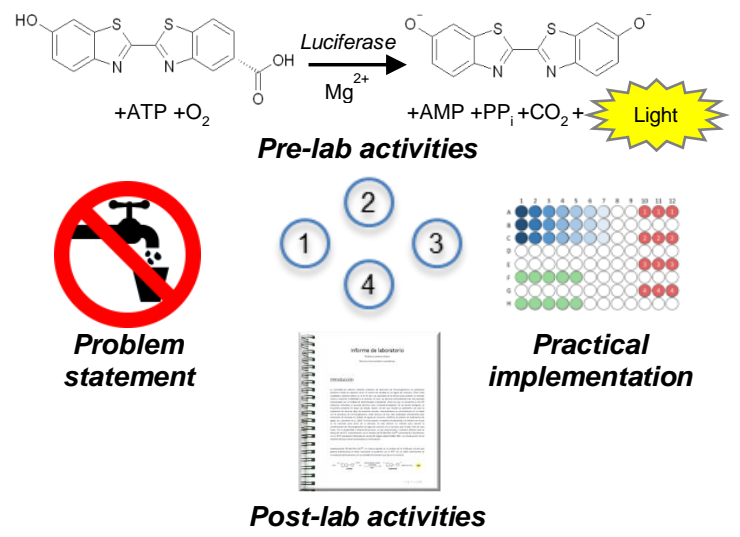

\section{KEYWORDS}

Upper-Division Undergraduate, Laboratory Instruction, Analytical Chemistry, Biochemistry, Hands-On Learning/Manipulatives, Problem Solving/Decision Making, Bioanalytical Chemistry, Quantitative Analysis, Water/Water Chemistry

Bioluminescence is a phenomenon that our students can easily identify in some animals, such as fireflies, which emit light to attract their mating partners. This light is the result of a chemical reaction catalyzed by the enzyme luciferase, which uses luciferin as a substrate and requires adenosine triphosphate (ATP), oxygen and a metal cation for its completion. Probably the most widely used luciferase is that of the firefly Photinus pyralis (EC 1.13.12.7), already described around the middle of the 20th century. ${ }^{1}$ The typical emission spectrum of this reaction (see Figure 1) is in the green-yellow region $(550-570 \mathrm{~nm})$, with a peak at $562 \mathrm{~nm}$ in the basic medium $(\mathrm{pH} \sim 7.5-7.8)^{2,3}$.

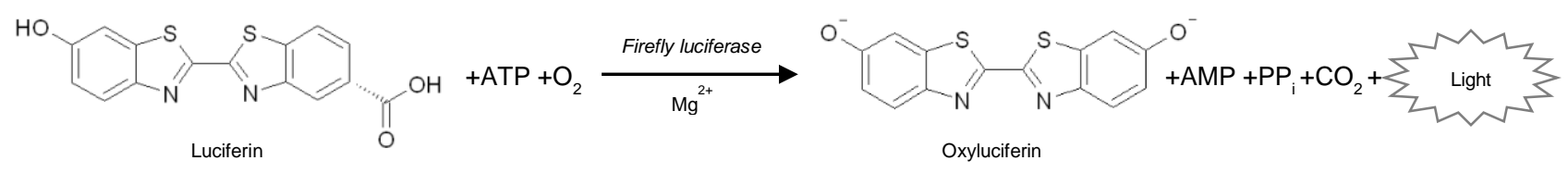

Figure 1. Luciferase-catalyzed enzymatic reaction

The practical applications of luciferases have been fueled by their isolation and purification from various species and by an efficient production of recombinant enzymes. The current availability of a number of commercial kits has made their use for a wide variety of applications cheaper, easier and more widespread. Some examples are the quantification of the luciferase-catalyzed reaction cofactors and the measurement of some luciferin-releasing enzymatic activities, including some proteases such 
as caspases, involved in apoptosis. In addition, luciferase is widely used for the in vitro and in vivo monitoring of biological processes, allowing the measurement of enzymatic activities, gene expression, protein-protein interactions and disease progression, with relevant clinical, diagnostic and drugdiscovery applications..$^{4,5}$

Since the early discovery that the light intensity emitted by the luciferase reaction is proportional to the ATP concentration in the analyzed sample, ${ }^{1}$ ATP quantification has been one of the most common applications of bioluminescence. Often described as the "energy currency" of biological cells, ATP is an excellent bioindicator of the presence of any type of living organism. ${ }^{6}$ The determination of ATP concentrations, based on the luciferase-catalyzed reaction is the most sensitive, simple and widely used for the detection of microbiological contamination in food industries, clinical diagnostics, etc. ${ }^{7}$ The direct correlation of ATP concentration and bacteria content is the basis for the development of rapid methods of enzymatic analysis that allow the assessment of bacterial contamination in drinking and water or in reservoirs, skipping the long delays required in traditional microbiological methods..$^{8,9}$

Throughout the last two years, we have implemented at the University of Malaga (Spain) a laboratory experiment that, aiming at the evaluation of microbial contaminations in water, illustrates in the framework of enzymatic analysis the principles and applications of bioluminescence to science undergraduate students. By using an affordable commercial reagent, bacteria are lysed, their ATP released and then quantified by means of the reaction catalyzed by a recombinant thermostable luciferase. ${ }^{10}$ This experiment was initially designed to be completed in a single laboratory session and was carried out by some hundred undergraduate students within the subject "Applied Biochemistry" taught during the third and fourth year of the biochemistry and chemistry BS degrees respectively.. After the successful implementation of this "short protocol", the aforementioned laboratory experiment has been adapted to a problem-based learning (PBL) format and tested with 4th year biochemistry/biotechnology students within the course "Advanced Instrumental Techniques". In this new format, the scope of this practical experience was not limited to teaching luminometry, also allowing the acquisition of some other transversal skills by students in the last years of their studies.

There is evidence that graduates in experimental sciences lack certain general skills that may be useful in their future professional development, including autonomous learning, planning of experiments, time and resource management, or the ability to present in public and work as a team. 
These flaws are identified by the students themselves when, after completing their studies and entering the labor market, they reflect on the strengths and weaknesses of their education at the university level. ${ }^{11}$ To a large extent, these shortcomings could be derived from the type of teaching most frequent in our universities, and in particular with regard to laboratory experiments, where students usually limit themselves to follow a procedure that has been predetermined by the teacher. ${ }^{12}$

As opposed to this "cookbook" approach, there are other practical science teaching styles that may bring students closer to the scientific method, encouraging them to ask questions, discuss protocols, take and analyze results, thus enabling them to draw conclusions with the support of the specialized literature. ${ }^{13,14}$ Course-based undergraduate research experiences (CUREs) ${ }^{15-17}$ and PBL approaches ${ }^{18-20}$ are methods successfully used to create a more enriching research experience for students. Extending from several weeks to a complete course, they are gradually becoming established in the practical teaching of chemistry and biochemistry undergraduate students, with an increase in their motivation..$^{21,22}$

Following this line of thought, in the PBL experience here presented, the laboratory work is just a part of a more complete practical project, developed throughout a full period. Starting with a real-life problem, students are driven through the process of searching for information and designing protocols. They are trained in operational issues such as the acquisition of reagents, method finetuning and experimental validation in the laboratory, and finally in the critical discussion of the results. This way students replicate situations they will most probably have to face in their next professional development, when they will have to employ their knowledge to solve specific problems. This new approach is aimed to awaken the interest of students who, being in the last stage of their studies, are particularly concerned about an increasingly demanding job market.

Finally, the modular nature of the PBL activities allows the individual and immediate application of some of these modules in lower level biochemistry and chemistry courses, taught as practical laboratory sessions under the traditional model. The application in our classrooms of both the extended and short protocols has been very gratifying, as evidenced by the good academic performance and the positive students' perception. 


\section{LEARNING OBJETIVES}

Learning goals for these two versions of the laboratory experiment are listed in Table 1. Generally, by measuring ATP concentrations in water, students gain knowledge about the principles and applications of bioluminescence and the practical use of luminometers. This is already valuable in itself because, as mentioned, luminometry is a neglected technique in our classrooms, particularly in terms of laboratory experiments availability. As expected, the learning goals for the PBL format include those from the short protocol, and others derived from the more active role played by students in their learning process, making them undertake authentic real-world tasks, similar to those they will find in their professional future. Thus, many of these students will face for the first time common issues for a professional, such as the acquisition of reagents and materials, the assessment of the necessary instruments and equipment, the adaptation and scaling of experimental protocols, or the analysis of costs and operational feasibility, among others.

\section{Table 1. Learning objectives for the two versions of this laboratory experiment}

\begin{tabular}{|c|c|c|}
\hline Learning Goals & PBL & Short Protocol \\
\hline 1. To search, apply and reference scientific literature & $\mathrm{X}$ & 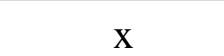 \\
\hline 2. To elaborate a laboratory protocol & $\mathrm{x}$ & \\
\hline 3. To detail material, reagents and instrumentation needed & $\mathrm{x}$ & \\
\hline 4. To make calculations and prepare solutions and reagents & $\mathrm{x}$ & \\
\hline 5. To use a luminometer and apply bioluminometry to a real-world problem & $\mathrm{X}$ & $\mathrm{X}$ \\
\hline 6. To know the principles and applications of bioluminometry & $\mathrm{x}$ & $\mathrm{X}$ \\
\hline 7. To set a calibration curve and establish the method application range & $\mathrm{x}$ & $X$ \\
\hline 8. To determine the limit of detection of a method & $\mathrm{x}$ & \\
\hline 9. To determine the ATP concentration of a sample & $\mathrm{x}$ & $\mathrm{x}$ \\
\hline 10. To look for information in order to propose methodological improvements. & $\mathrm{x}$ & $\mathrm{x}$ \\
\hline 11. To determine which method is the most suitable for a given real-world problem & $\mathrm{x}$ & \\
\hline 12. To compare and contrast luminometry with fluorometry and spectrophotometry & $\mathrm{x}$ & \\
\hline 13. To present their results and discuss them based on primary literature & $\mathrm{x}$ & $\mathrm{X}$ \\
\hline 14. To write a scientific journal-quality report & $\mathrm{x}$ & \\
\hline 15. To face challenges in a real-world scenario & $\mathrm{x}$ & \\
\hline 16. To present results in front of a diverse audience & $\mathrm{x}$ & \\
\hline 17. To summarize conclusions in an "executive inform" & $\mathrm{X}$ & \\
\hline
\end{tabular}




\section{EXPERIMENTAL OVERVIEW}

Students, working in groups of $4-5$, carry out independently the activities detailed below, under the guidance of the responsible teacher in the role of the facilitator. The PBL activity flow chart, developed over a period of 4-6 weeks according to the subject scheduling requirements, is illustrated in Figure 2.
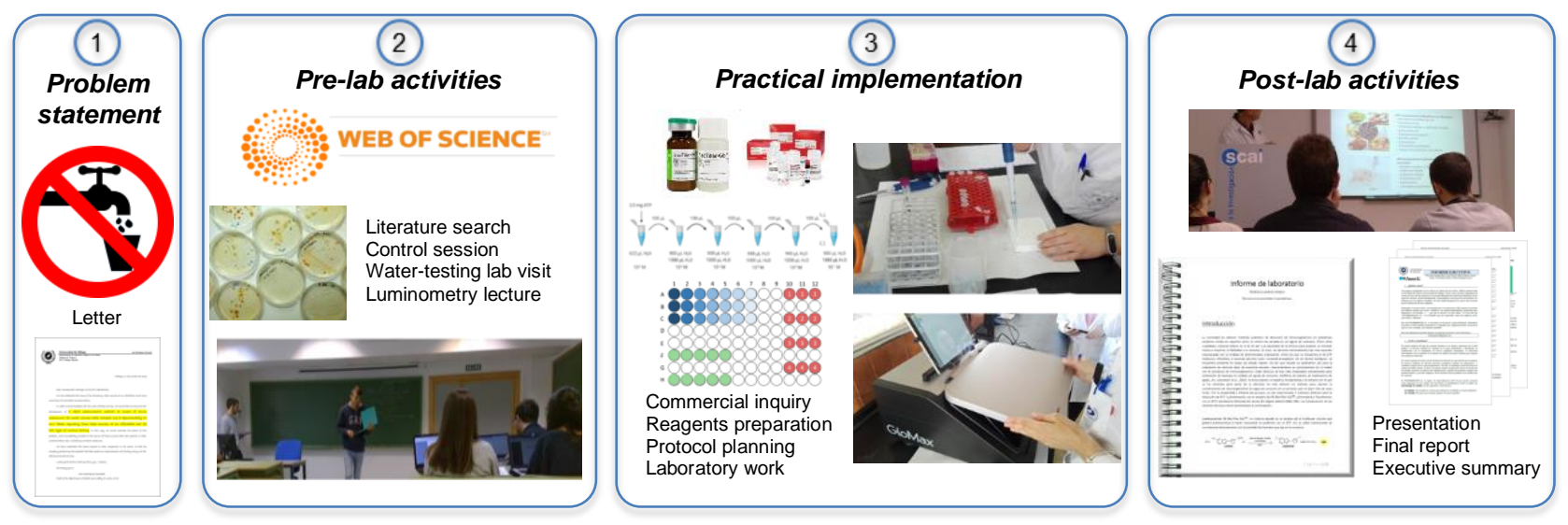

Figure 2. Flow chart of the problem based learning experience.

1. Problem statement: In the first lecture, the teacher introduces the PBL activity, asking students to split into groups (4-5 students each), which will act as "corporations" that must offer a solution for a given technical issue that has to be resolved throughout the course. Each company receives a letter (see Box 1) exposing the problem (implement a method for rapid determination of microbial contamination in water). In that letter, the main requisites of the desired analytical method are presented: quickness (results must be available in a few hours), affordability (a high number of water samples should be routinely assayed) and sensibility (sample volumes should be restricted to a few milliliters). 


\section{Box 1. Problem Statement letter. The main characteristics of the required analytical method have been highlighted in yellow.}

Dear commercial Manager of $x x x x$ Laboratories.
we have detected that some of the drinking water fountains at
our facilities could have some kind of microbial contamination.
In order to avoid health problems, we would like to outsource the
development of a rapid measurement method (a couple of hours,
maximum) for small volume water samples (up to
approximately 10 mL) taken regularly from these
fountains, at an affordable cost for this type of routine
testing. In this way, we would evaluate the extent of this problem, and
immediately proceed to the closure of those water fountains that were positive
in their contamination test, initiating corrective measures.
we have submitted this same request to other companies in the area, so that
the one presenting the proposal that best meets our requirements will finally
carry out the aforementioned services.
Looking forward to hearing from you, I remain.

2. Pre-lab activities.

a. Literature search: Students, complete bibliographic research with the purpose of finding methods that could provide an answer to the established problem. They write a first report in which they summarize their findings and critically expose pros and cons of each method. Furthermore, they must evaluate their suitability taking into account time requirements, cost of the assay and instrumentation, amount of sample needed, etc.

Deliverable: Bibliography search report. Autonomous work of the student, working in small groups. Due: 2 days before the classroom control session.

b. Classroom control session: All groups share their bibliographic reviews. They present their findings in a session in which the teacher, acting as a moderator, can check the students' search for solutions. After this session, it should be clear to the students that they must use "chemical" 
methods that do not require several days for their completion, unlike traditional microbiological methods.

Deliverable: Bibliography search presentation in class.

c. Visit to water testing facilities: Students visit facilities that perform microbiological cultures for water routine analyses. They comprehend that these "traditional" methods, based in the in vitro growing of the microorganisms, are not a practical solution to the given issue.

d. Lecture: In a one-hour lecture, the teacher summarizes some key principles and applications of bioluminometry (an index of the topics covered in this lecture is presented as supplementary material).

3. Practical implementation: Once the possible solutions to the problem are identified (mainly methods based on the enzymatic measurement of ATP), students carry out the necessary steps for their subsequent practical implementation:

a. Commercial inquiry: Identify which reagents and instrumentation are required. Design of the experimental protocols and calculations needed to prepare the reagents.

Deliverable: A report per group. Autonomous work of the student, working in small groups, supervised by the teacher. Due: one week before the laboratory sessions.

b. Laboratory work (as demanded by students, typically two 3-4 hours sessions): Preparation of Reagents and solutions preparation. Implementation of the protocols to measure ATP concentration by spectrophotometry, fluorimetry, and luminometry. Data are obtained for the calibration curves drawing and the methods comparison (detection limit, accuracy...). Water samples from different sources are analyzed.

Autonomous work of the student, in small groups, supervised and evaluated by the teacher.

4. Post-lab activities:

a. Writing of a final report in which, based on the comparative study of the results obtained with the three methods, students will:

- Discuss its applicability to the problem initially raised.

- Make proposals to improve and optimize the protocols.

- Estimate cost of the assays. 
Deliverable: Individual final report. Autonomous work of the students, corrected and evaluated by the teacher. Due: 2-3 weeks after the end of the practical implementation, at least one week before the final session.

b. Final session: Presentation of the results obtained by the different groups, replicating a work corporate meeting (group session, facilitated by the teacher). Deliverable: Presentation. Autonomous work of the student, working in small groups, supervised by the teacher. Due: the presentation is given to the teacher 2 days before this session.

c. Drafting of an executive summary in response of statement letter.

Deliverable: Executive summary. Small group. Due: 2 days before the final session.

Components of the short laboratory experiment

1. Pre-lab activity: Lecture: In a one-hour lecture, the teacher summarizes some key principles and applications of bioluminometry.

2. Laboratory work (1-2 hours): Students follow the protocol (students and instructor protocols are available as supplementary material) to measure ATP concentrations by using luciferase. They get results to draw a calibration curve and analyze water samples from different sources. Autonomous work of the student, supervised by the teacher. Groups of 20-30 students, working in pairs.

3. Post-lab activity: Writing of a final report in which students draw the calibration curve and determine the water samples ATP concentration. They discuss the applicability of the method to the problem initially raised and discuss the pros and cons compared to traditional methods for detecting water pollution based on microbiological techniques. They suggest, based on bibliography, some improvements that could help to increase the method sensitivity, and to distinguish between the water-dissolved ATP and that contained in the bacteria.

Deliverable: Individual final report. Autonomous work of the student, corrected and evaluated by the teacher. 


\section{STUDENTS' RESULTS}

PBL students' results

This experience was completed in the 2019-20 course by ten 4th year biochemistry/biotechnology students enrolled in the subject "Advances Instrumental Techniques". They split into two groups of 5 and, after receiving the letter stating the analytical problem (see Box 1), they searched the literature for possible solutions. In an initial report, each group presented different possible ways of measuring biological contamination in water, classifying them according to the techniques used. Criteria such as test length, sensitivity and cost led them to focus on those measuring ATP concentration (see Figure 3).

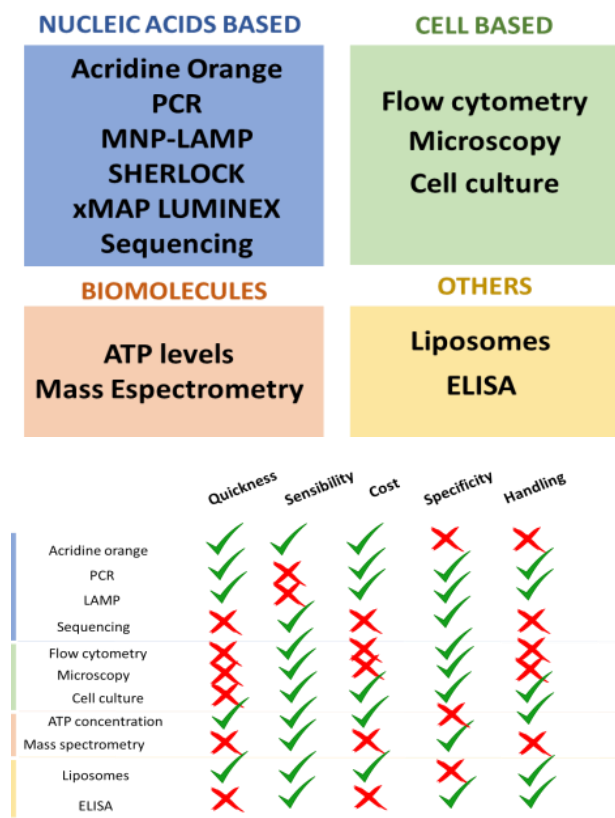

Figure 3. Methods comparison reported by one of the groups after the literature search

All this information would be sufficient to move on to the next stage, i.e. the implementation of the chosen methods in the laboratory. However, we included a control session, in which both groups presented the results of their bibliographic research and their conclusions. This allowed the teacher to redirect the work of any group that might be left behind at this point and also unify the methods to be later implemented, in order to facilitate practical operations and minimize costs by the use of common reagents.

In addition, the teacher could contribute to outline the methods to be later implemented by the students. For example, in our case, although the two groups proposed a similar method for the measurement of ATP by means of luciferase ${ }^{23}$, only one of the groups mentioned the improvement of 
this method reported by Hammnes et al. $2010^{10}$, which increases sensitivity in several orders of magnitude. Thanks to this feedback session, both groups were able to plan their future tasks with the highest success guarantees.

The students also proposed to carry out the enzymatic measurement of ATP by spectrophotometric and fluorometric methods. In order to unify and simplify their laboratory work, we agreed to use a commercial kit that enabled the use of both determination techniques in a single assay ${ }^{24}$.

At this point, the groups were able to organize their work independently, requesting materials, instrumentation and the laboratory bench to perform their experiments (the protocols used by the students are shown as supplementary material). The instructor reviewed the experimental planning and accompanied the students in these practical laboratory sessions, acting as a mere observer and only intervening when necessary, as for example, to explain how to use the luminometer.

The students obtained the necessary data to draw the calibration lines for the spectrophotometric, fluorometric and luminometric methods (Figure 4, upper, middle and lower, respectively), adapting the concentration ranges to what was indicated in the bibliography or in the instructions of the reagent suppliers. In addition, they analyzed three water samples provided by the instructor: Chlorinated tap water (sample A), water from an undrinkable decorative fountain (sample B), and a solution of ATP (1 $\mathrm{nM}$ ), used by the teacher to evaluate the students' accuracy (sample C). In the case of the luminiscent measurement, they started using ATP concentrations ranging from $10^{-12}$ to $10^{-7} \mathrm{M}$ to estimate the water samples ATP concentrations, and then repeated the assay focusing on the lower concentrations, in order to optimize the analysis results (Figure 4, lower panel). 

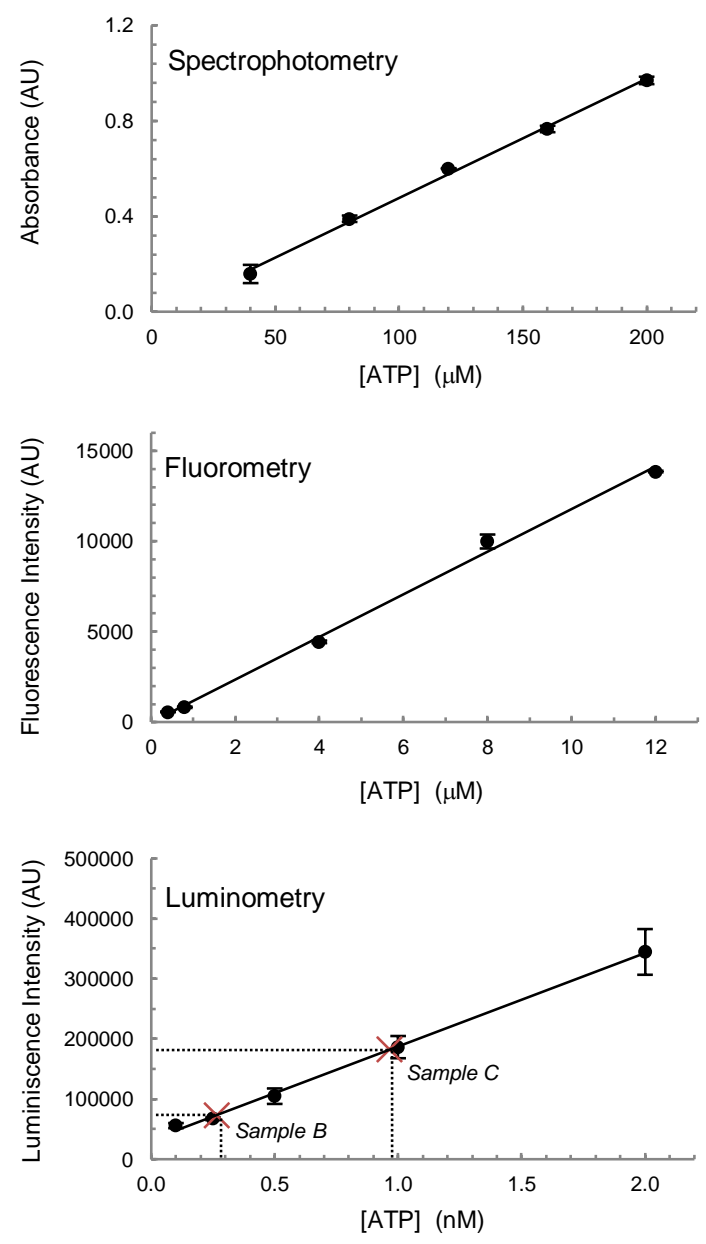

Figure 4. Results obtained by one the groups performing the PBL experience. Calibration curves for spectrophotometric (upper), fluorometric (middle) and luminometric (lower) methods. Data in curve are mean + S.D., $n=3$. Interpolation of water samples $B$ and $C$ in the luminometry calibration line is shown (sample A value was too low to be interpolated)

With regard to the problem water samples, students could calculate the ATP concentrations of

samples $\mathrm{B}$ and $\mathrm{C}$ by interpolation of their luminescence intensity values in the luminometry calibration line. They identified them as two putative "undrinkable fountains", whereas the low luminiscente intensity value obtained for sample A (1200 AU), suggested that this water fountain was drinkable. They didn't know it, but sample C allowed the teacher to evaluate their accuracy, which was higher than $98 \%$ for both groups. They also concluded that only luminometry provided the sensitivity needed for the detection of microbiological contamination in the supplied water samples, since values of absorbance (ranging from 0.042 to $0.050 \mathrm{AU}$ ) or fluorescence intensity (ranging from 353 to $434 \mathrm{AU}$ ) for the 3 water samples were too low to be interpolated in the spectrophotometry or the fluorometry calibration curves. 
To further estimate the suitability or not of each method to determine bacterial contaminations, the students determined their limits of detection (LOD) and quantification (LOQ). LOD of an analytical method, or the lowest analyte concentration at which detection is feasible, can be statistically calculated as the mean blank value plus 3 SDs. LOQ, or the lowest concentration at which the analyte can be quantified at a given confidence level, is often calculated as the mean blank value plus 10 SDs. ${ }^{25,26}$ Students analyzed a series of 10 identical blank (negative) samples that were used to calculate the means and SD for the three analytical methods. Their results (Table 2) evidenced that the sensibility for the three enzymatic analysis methods to quantify ATP concentrations could be ordered as such: luminometry>fluorometry>spectrophotometry. Since the LOD obtained for the luminometric method was two orders of magnitude higher than that reported by Hammes ${ }^{10}$, students suggested that there was room for improvement, probably by better controlling the incubation temperature.

\begin{tabular}{|c|c|c|}
\hline Methods & $L O Q(M)^{a}$ & $L O D(M)^{b}$ \\
\hline Spectrophotometry & $7.0 \cdot 10^{-6}$ & $4.3 \cdot 10^{-6}$ \\
\hline Fluorometry & $9.3 \cdot 10^{-7}$ & $6.8 \cdot 10^{-7}$ \\
\hline Luminometry & $3.3 \cdot 10^{-10}$ & $1.4 \cdot 10^{-10}$ \\
\hline \multicolumn{3}{|c|}{$\begin{array}{l}a \text { Limit of detection (LOD) and } b \text { Limit of quantification } \\
\text { (LOQ) for the three ATP analytical methods. Data } \\
\text { reported by one students' group. }\end{array}$} \\
\hline
\end{tabular}

Despite working in the laboratory in small groups, students had to write a final individual report. That allowed a more individualized evaluation of their individual work, confronting them with the drafting of the discussion on the results, as well as with the bibliographic review. An outline of the final report is supplied as supplementary material.

As an example of commentaries included in this final report, Figure 5 shows the proposal presented by one of the students after some bibliographic research, ${ }^{27,28}$ regarding the putative enzymatic reactions and reagents used in the spectrophotometric / fluorometric assay (this information was not provided by the supplier) 


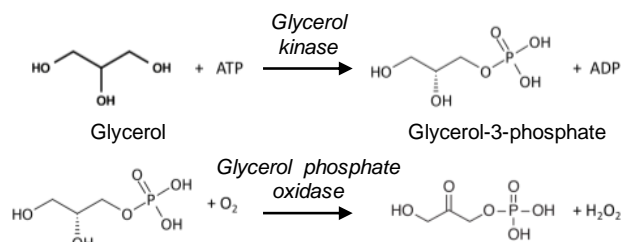

Glycerol-3-phosphate Dihydroxiacetone phosphate

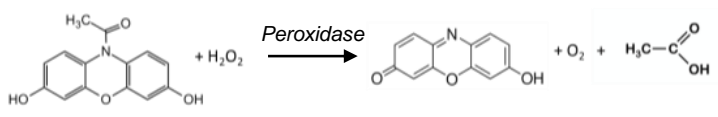

10-Acetyl-3,7-dihydroxyphenoxazine Resorufin

275 Figure 5. Coupled enzymatic reactions for the spectrophotometric and/or fluorometric determination of ATP, as proposed by one of the students.

In the final session the students presented their results and conclusions, after summarizing them in form of a short executive report following the teacher's indications (see supplementary material).

Short protocol students' results

Firstly performed by about forty 4th year chemistry students in the 2018-19 course, this protocol was carried out, in its final form, by sixty 3rdyear biochemistry students in the 2019-20 course, within the "Applied Biochemistry" subject.

Figure 6 shows a calibration curve obtained by one of those 3rd year biochemistry students. As observed, the application range reached as low as $[\mathrm{ATP}]=10^{-12} \mathrm{M}$, probably because the pre-warming of samples and reagents was well controlled under the instructor's supervision. Most students were able to identify that only sample A (with a luminescence intensity value close to that of the blank) was drinkable. Sample C results (1 nM ATP solution), were used by the teacher to evaluate the students' accuracy.

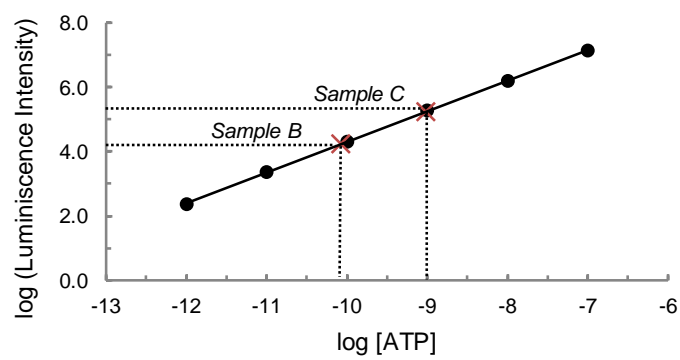

Figure 6. Calibration curve obtained by one of the students following the short protocol. Data in curve are mean of duplicates. Interpolation of water samples $B$ and $C$ is shown (sample $A$ value was too low to be interpolated)

\section{STUDENTS' ACHIEVEMENT OF THE LEARNING GOALS}

A first estimation of the students' progress was made by means of an assessment test composed by eight short open-questions (see Figure 7). This test was administered at the beginning of the 
introductory lecture (pretest) and repeated after the full reports were delivered by the students (posttest). As observed in Figure 7, an improvement in the students' knowledge about bioluminescence and the applications and fundamentals of luminometry was achieved after performing either the PBL or the short protocol.
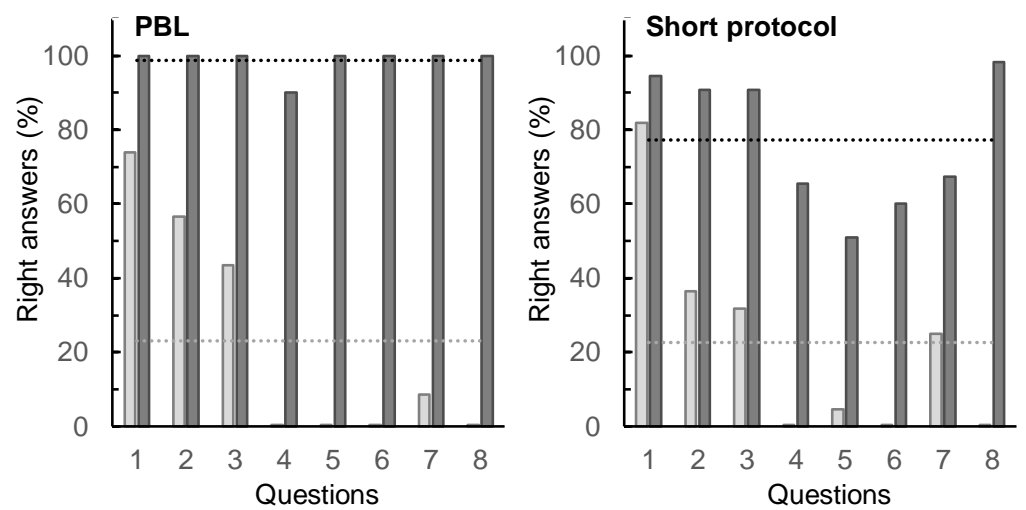

$\square$ Pretest

$\square$ Posttest

Pretest average
1. What do fluorescence and luminescence have in common?

2. How are they different?

3. What do a fluorimeter and a

luminometer have in common?

4. How are they different?

5. Could you indicate the substrates, effectors, products of the luciferase catalyzed reaction?

6. How are luciferases classified according to their kinetics?

7. What applications of luminometry do you remember?

8. How would you measure the presence of bacterial contamination in a sample?

Figure 7. Student's outcome evaluation. Comparison of the percentage of students who answered correctly the pre and posttest questions. Left. PBL students; Middle. Short protocol students; Right. Questions included in the pre- and posttest questionnaire.

Although initially many students were able to identify fluorescence and chemiluminescence as two light-emitting phenomena, it was only after performing the experiment that they were clear about their differences, as well as the characteristics of the instrumentation used by those methods. In the pretest, most of the students were not able to recall the components of the luciferase reaction (substrates, cofactors, products) as well as the kinetic characteristics of these enzymes, which allow their application in flash or glow mode. In the posttest their knowledge of these issues increased, with the PBL students internalizing this content much better than those who carried out the short protocol. As far as the applications of luminometry are concerned, although initially the students' answers were mostly limited to the use of luminol in forensic medicine, after their work in the laboratory, practically all of them were able to mention several others applications and could describe how to use the ATP measurement to detect bacterial contamination.

Students' scores, based on their achievement of the learning goals (LG, detailed in Table 1), were assessed by application of the corresponding evaluation rubrics (see supplementary material). As can be seen in Figure 8, the learning results were satisfactory, with most of students knowing the luminometry principles, applications and instrumentation (LG\#5 and 6). Nevertheless, it is quite clear 
that the PBL method obtained better and more extensive results. In addition to achieving a higher number of learning goals, derived from the more comprehensive nature of their learning experience, students also obtained better scores in those LGs shared with students performing short protocol.

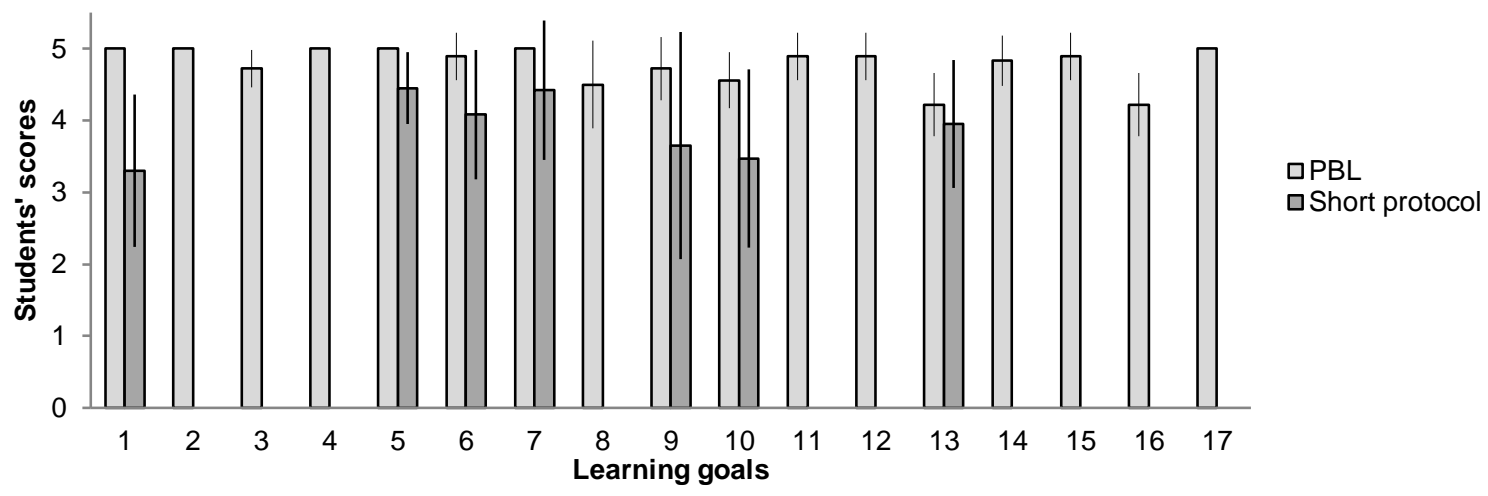

Figure 8. Students' achievement of the learning goals presented in Table 1. Students' scores were assessed by application of the corresponding evaluation rubrics, ranging from 0 (absent) to 5 (excellent). Data are presented as mean $\pm S D$ ( $n=10$ for PBL and $n=60$ for short protocol)

In particular, great differences were observed in those tasks that required critical discussion of the results and the proposal of improvements according to the literature (LG\#1 and 10). In addition, it was found that although, in general, students performed the protocol correctly and could draw valid calibration curves (LG\#7), some of those performing the short protocol made mistakes when they had to calculate the concentration of the problem samples (LG\#9), which did not occur with those who followed the PBL.

\section{STUDENTS' FEEDBACK}

Students' perception of the teaching methodology used in the new PBL experience was evaluated by means of a post course mixed questionnaire, using some 1 to 5 Likert-type scale questions, complemented with some other open-answered to collect anonymous comments from the students. Results, shown as supplementary material, indicate that students had a very positive perception to the whole practical sequence, by scoring it an average of 4.7 out of 5 .

Among other aspects, they noticed the novelty of the learning methodology (PBL) used in this activity with respect to that used in other subjects of their degree. They considered that they had learnt the luminometry fundamentals and instrumentation, knowing some applications of the bioluminescence measurement. In addition, they acknowledged that with this PBL experience they understood how to best develop a laboratory protocol and to establish a work plan, contrasting with 
other practical lessons they had carried out previously. Although it had required more work and preparation than other laboratory experiments carried out throughout their undergraduate studies, they considered that this experince had been worthwhile because they had learned more. They felt that it had also allowed them to approach situations that could be similar to those found in their professional future. Moreover, they appreciated being able to seek solutions through bibliographical research, as well as planning and organizing the tasks to be carried out in the laboratory.

These result are in agreement with the previous observation that although inquiry-based approaches normally take more effort and time to develop, they are usually very positively perceived by students, who become more actively involved in their learning process and find this type of research experience very rewarding. ${ }^{29-31}$ At a time when graduate employability is a key issue for higher education, as new graduates are faced with a highly competitive and rapidly evolving employment landscape, a shift from more traditional teaching systems to other thought-provoking learning approaches will help promote those skills and attributes that are most valued by employers. ${ }^{22,32}$

HAZARDS

Students should take common precautions for chemical operations laboratory, minimizing the risk of ingestion, inhalation or contact. They should also wear appropriate personal protective equipment (lab coat, gloves and safety goggles). The BacTiter-Glo ${ }^{\mathrm{TM}}$ Substrate and Buffer are not classified as hazardous according to the Globally Harmonized System (GHS). ATP Colorimetric/Fluorometric Assay Kit is not a hazardous substance or mixture according to Regulation (EC) No. 1272/2008. More information is available in Notes for Instructors (Supplemental material).

\section{SUMMARY}

ATP quantification by measuring the light generated by the luciferase-catalyzed reaction has been successfully applied to teach the use of bioluminescence to science undergraduate students. The statement of an authentic driving question, as is the detection of microbiological contaminations in water, increased the students' interest to find answers, collaborate with each other and learn new methodologies. In both formats used, either as a project developed by students throughout the full course or as a short protocol carried out in a single laboratory session, the students' achievement of the learning goals was very satisfactory. Those students who followed the PBL approach, and therefore took a more active role in their learning, could better assimilate the knowledge, and, in addition, 
adopted a series of transversal skills that they recognized would be useful in their future professional ventures.

\section{ASSOCIATED CONTENT}

Supporting Information

The Supporting Information is available on the ACS Publications website at DOI:

10.1021/acs.jchemed.XXXXXXX. [ACS will fill this in.] Example brief descriptions with file formats indicated are shown below; customize for your material.

Index of the topics covered in the luminometry lecture (.pdf)

Short Protocol (.pdf):

Students' material: Laboratory protocol

Notes for instructors: Instructor laboratory protocol

Evaluation rubric and students' achievement of the learning goals

Problem Based Learning experience (.pdf):

Statement letter

Instructions for students

Students's protocols (a brief example from a group of students)

Evaluation rubrics and students' achievement of the learning goals

Students' perception: Likert questionnaire, including results obtained and some students' commentaries.

\section{AUTHOR INFORMATION}

Corresponding Author

*E-mail: quesada@uma.es

ORCID

Ana R. Quesada: 0000-0002-6419-8867

$395 \quad$ Notes

The authors declare no competing financial interest.

\section{ACKNOWLEDGMENTS}

This work was supported by the University of Malaga (Spain) funds granted to the educational innovation projects PIE19-086 \& PIE19-057, and the Spanish Ministry of Science, Innovation and Universities grant EDU2017-82197-P. The help of the Biochemistry and Chemistry undergraduate students of University of Malaga in performing experiments and collecting data is greatly appreciated. 


\section{REFERENCES}

1. McElroy, W. D. The Energy Source for Bioluminescence in an Isolated System. Proc. Natl. Acad. Sci. U. S. A. 1947, 33 (11), 342-345. DOI: $10.1073 /$ pnas.33.11.342

2. Seliger, H. H.; Buck, J. B.; Fastie, W. G.; McElroy, W. D. The Spectral Distribution of Firefly Light. J. Gen. Physiol. 1964, 48 (1), 95-104. DOI: 10.1085/jgp.48.1.95

3. Marques, S. M.; Esteves da Silva, J. C. G. Firefly Bioluminescence: A Mechanistic Approach of Luciferase Catalyzed Reactions. IUBMB Life. 2009, 61 (1), 6-17. DOI: 10.1002/iub.134

4. Roda, A.; Pasini, P.; Mirasoli, M.; Michelini, E.; Guardigli, M. Biotechnological Applications of Bioluminescence and Chemiluminescence. Trends Biotechnol. 2004, 22 (6), 295-303. DOI: 10.1016/j.tibtech.2004.03.011

5. Roda, A.; Guardigli, M.; Michelini, E.; Mirasoli, M. Bioluminescence in analytical chemistry and in vivo imaging. Trends Anal. Chem. 2009, 28 (3), 307-322. DOI: 10.1016/j.trac.2008.11.015

6. Hawronskyj, J. M.; Holah, J. ATP: A Universal Hygiene Monitor. Trends Food Sci. Technol. 1997, 8 (3), 79-84. DOI: 10.1016/S0924-2244(97)01009-1

7. Khlyntseva, S. V.; Bazel', Ya. R.; Vishnikin, A. B.; Andruch, V. Methods for the Determination of Adenosine Triphosphate and Other Adenine Nucleotides. J. Anal. Chem. 2009, 64 (7), 657-673. DOI: $10.1134 / \mathrm{S} 1061934809070028$

8. Delahaye, E.; Welté, B.; Levi, Y.; Leblon, G.; Montiel, A. An ATP-Based Method for Monitoring the Microbiological Drinking Water Quality in a Distribution Network. Water Res. 2003, 37 (15), 3689-3696. DOI: 10.1016/S0043-1354(03)00288-4

9. Eydal, H. S. C.; Pedersen, K. Use of an ATP Assay to Determine Viable Microbial Biomass in Fennoscandian Shield Groundwater from Depths of 3-1000 m. J. Microbiol. Methods 2007, 70 (2), 363-373. DOI: 10.1016/j.mimet.2007.05.012

10. Hammes, F.; Goldschmidt, F.; Vital, M.; Wang, Y.; Egli, T. Measurement and Interpretation of Microbial Adenosine Tri-Phosphate (ATP) in Aquatic Environments. Water Res. 2010, 44 (13), 3915-3923. DOI: $10.1016 / j$.watres.2010.04.015

11. Hanson, S.; Overton, T. Skills Required by New Chemistry Graduates and Their Development in Degree Programmes. Hull: The Higher Education Academy UK Physical Sciences Centre, UK, 2010. http://www.rsc.org/learn-chemistry/resources/business-skills-and-commercialawareness-for-chemists/docs/skillsdoc1.pdf

12. Domin, D. S. A Review of Laboratory Instruction Styles. J. Chem. Educ. 1999, 76 (4), 543-547. DOI: $10.1021 /$ ed076p543

13. National Research Council. Inquiry and the National Science Education Standards: A guide for Teaching and Learning. The National Academies Press: Washington, DC, 2000. DOI: $10.17226 / 9596$ 
14. Weaver, G. C.; Russell, C. B.; Wink, D. J. Inquiry-Based and Research-Based Laboratory Pedagogies in Undergraduate Science. Nat. Chem. Biol. 2008, 4 (10), 577-580. DOI: 10.1038/nchembio1008-577

15. Clark, T. M.; Ricciardo, R.; Weaver, T. Transitioning from Expository Laboratory Experiments to Course-Based Undergraduate Research in General Chemistry. J. Chem. Educ. 2016, 93 (1), 56-63. DOI: 10.1021/acs.jchemed.5b00371

16. Williams, L. C.; Reddish, M. J. Integrating Primary Research into the Teaching Lab: Benefits and Impacts of a One-Semester CURE for Physical Chemistry. J. Chem. Educ. 2018, 95 (6), 928-938. DOI: $10.1021 /$ acs.jchemed.7b00855

17. Marsiglia, W. M.; Qamra, R.; Jackson, K. M.; Traaseth, N. J. A CURE Biochemistry Laboratory Module to Study Protein-Protein Interactions by NMR Spectroscopy. J. Chem. Educ. 2020, 97 (2), 437-442. DOI: 10.1021/acs.jchemed.9b00364

18. Budner, D.; Simpson, B. Project-Based Integrated Lecture and Laboratory Quantitative Analysis Course. J. Chem. Educ. 2018, 95 (9), 1533-1540. DOI: 10.1021/acs.jchemed.8b00146

19. Diawati, C.; Agus, L.; Buchari, S. Using Project-Based Learning To Design, Build, and Test Student-Made Photometer by Measuring the Unknown Concentration of Colored Substances. J. Chem. Educ. 2018, 95 (3), 468-475. DOI: 10.1021/acs.jchemed.7b00254

20. Hensen, C.; Clare, T. L.; Barbera, J. Using Quenching To Detect Corrosion on Sculptural Metalwork: A Real-World Application of Fluorescence Spectroscopy. J. Chem. Educ. 2018, 95 (5), 858-863. DOI: $10.1021 /$ acs.jchemed.7b00726

21. Irby, S. M.; Pelaez, N. J.; Anderson, T. R. Student Perceptions of Their Gains in Course-Based Undergraduate Research Abilities Identified as the Anticipated Learning Outcomes for a Biochemistry CURE. J. Chem. Educ. 2020, 97 (1), 56-65. DOI: 10.1021 /acs.jchemed.9b00440

22. Nagarajan, S.; Overton, T. Promoting Systems Thinking Using Project- and Problem-Based Learning. J. Chem. Educ. 2019, 96 (12), 2901-2909. DOI: 10.1021/acs.jchemed.9b00358

23. Promega. https://www.promega.com/products/cell-health-assays/cell-viability-and-cytotoxicityassays/bactiter_glo-microbial-cell-viability-assay/?catNum=G8230 (accessed May 2020).

24. Merck. https://www.sigmaaldrich.com/catalog/product/sigma/mak190?lang=en\&region=US (accessed May 2020).

25. Armbruster, D. A.; Tillman, M. D.; Hubbs, L. M. Limit of Detection (LOD)/Limit of Quantitation (LOQ): Comparison of the Empirical and Statistical Methods Exemplified with GC-MS Assays of Abused Drugs. Clin.Chem. 1994, 40 (7), 1233-1238. DOI: 10.1093/clinchem/40.7.1233

26. Tholen, D. W.; Linnet, K.; Kondratovich, M. V.; Armbruster, D. A.; Garret, P.; Jones, R. L.; Kroll, M. H.; Lequin, R. M.; Pankratz, T.; Scassellati, G. A.; Schimmel, H.; Tsai, J. Protocols for Determination of Limits of Detection and Limits of Quantitation; Approved Guideline; NCCLS Document EP17-A. NCCLS: Wayne, PA, 2004.

https://www.semanticscholar.org/paper/Protocols-for-Determination-of-Limits-of-DetectionTholen-Linnet/9afc36c690cfbf76f9251464045564a5ee073d7b 
27. Fossati, P.; Prencipe, L. Serum Triglycerides Determined Colorimetrically with an Enzyme that Produces Hydrogen Peroxide. Clin. Chem. 1982, 28 (10), 2077-2080. DOI:

10.1093/clinchem/28.10.2077

28. Zhou, M.; Diwu, Z.; Panchuk-Voloshina, N.; Haugland, R. P. A Stable Nonfluorescent Derivative of Resorufin for the Fluorometric Determination of Trace Hydrogen Peroxide: Applications in Detecting the Activity of Phagocyte NADPH Oxidase and Other Oxidases. Anal. Biochem. 1997, 253 (2), 162-168. DOI: 10.1006/abio.1997.2391

29. Seymour, E.; Hunter, A. B.; Laursen, S. L.; DeAntoni, T. Establishing the Benefits of Research Experiences for Undergraduates in the Sciences: First Findings from a Three-Year Study. Sci. Educ. 2004, 88 (4), 493-534.DOI: 10.1002/sce.10131

30. George-Williams, S. R.; Soo, J. T.; Ziebell, A. L.; Thompson, C. D.; Overton, T. L. Inquiry and Industry Inspired Laboratories: The Impact on Students' Perceptions of Skill Development and Engagements. Chem. Educ. Res. Pract. 2018, 19 (2), 583-596. DOI: 10.1039/C7RP00233E

31. George-Williams, S. R.; Ziebell, A. L.; Thompson, C. D.; Overton, T. L. Inquiry-, Problem-, Context- and Industry- Based Laboratories: An Investigation into the Impact of Large-Scale, Longitudinal Redevelopment on Student Perceptions of Teaching Laboratories. Int. J. Sci. Educ. 2020, 42 (3), 451-468. DOI: 10.1080/09500693.2020.1714788

32. Sarkar, M.; Overton, T.; Thompson, C. D.; Rayner, G. Academics' Perspectives of the Teaching and Development of Generic Employability Skills in Science Curricula. High. Educ. Res. Dev. 2019, 39 (2), 346-361. DOI: 10.1080/07294360.2019.1664998 